\title{
BMJ Open Associations between lipid profiles of adolescents and their mothers based on a nationwide health and nutrition survey in South Korea
}

\author{
Ji Hyung Nam, ${ }^{1,2}$ Jaeyong Shin, ${ }^{3}$ Sung-In Jang, ${ }^{3}$ Ji Hyun Kim, ${ }^{4}$ Kyu-Tae Han, ${ }^{5}$ \\ Jun Kyu Lee, ${ }^{1}$ Yun Jeong Lim, ${ }^{1}$ Eun-Cheol Park ${ }^{\oplus}$
}

To cite: Nam JH, Shin J, Jang S-I, et al. Associations between lipid profiles of adolescents and their mothers based on a nationwide health and nutrition survey in South Korea. BMJ Open 2019;9:e024731. doi:10.1136/ bmjopen-2018-024731

- Prepublication history and additional material for this paper are available online. To view these files, please visit the journal online (http://dx.doi org/10.1136/bmjopen-2018024731).

Received 18 June 2018 Revised 23 January 2019 Accepted 23 January 2019

Check for updates

(C) Author(s) (or their employer(s)) 2019. Re-use permitted under CC BY-NC. No commercial re-use. See rights and permissions. Published by BMJ.

For numbered affiliations see end of article.

Correspondence to

Dr Eun-Cheol Park;

ecpark@yuhs.ac

\section{ABSTRACT}

Objectives Dyslipidaemia is a metabolic disease

influenced by environmental and genetic factors.

Especially, family history related to genetic background

is a strong risk factor of lipid abnormality. The aim of this study is to evaluate the association between the lipid profiles of adolescents and their mothers.

Design A cross-sectional study.

Setting The data were derived from the Korea National Health and Nutrition Examination Survey (IV-VI) between 2009 and 2015.

Participants 2884 adolescents aged $12-18$ years and their mothers were included.

Primary outcome measures Outcome variables were adolescents' lipid levels. Mothers' lipid levels were the interesting variables. The lipid profiles included total cholesterol (TC), triglyceride (TG), high-density lipoprotein cholesterol (HDL-C) and low-density lipoprotein cholesterol (LDL-C). We identified partial correlation coefficients ( $)$ between the lipids. Multiple linear regressions were performed to identify the amount of change in adolescents' lipid levels for each unit increase of their mothers' lipids. The regression models included various clinical characteristics and health behavioural factors of both adolescents and mothers.

Results The mean levels of adolescents' lipids were 156.6, 83.6, 50.4 and $89.4 \mathrm{mg} / \mathrm{dL}$, respectively for TC, TG, HDL-C and LDL-C. Positive correlations between lipid levels of adolescents and mothers were observed for TC, TG, HDL-C and LDL-C ( $r, 95 \%$ Cl: $0.271,0.236$ to 0.304 ; $0.204,0.169$ to $0.239 ; 0.289,0.255$ to 0.322 ; and 0.286 , 0.252 to 0.319 ). The adolescent TC level was increased by $0.23 \mathrm{mg} / \mathrm{dL}$ for each unit increase of the mother's TC (SE, 0.02; $p<0.001$ ). The beta coefficients were 0.16 (SE, 0.01), 0.24 (SE, 0.02) and 0.24 (SE, 0.02), respectively, in each model of TG, HDL-C and LDL-C (all $p<0.001$ ). The linear relationships were significant regardless of sex and mother's characteristics.

Conclusions Mothers' lipid levels are associated with adolescents' lipids; therefore, they can serve as a reference for the screening of adolescent's dyslipidaemia.

\section{INTRODUCTION}

Dyslipidaemia is a well-known risk factor for cardiovascular disease (CVD) in individuals of
Strengths and limitations of this study

- This study analysed the linear relationships between lipid profiles of adolescents and their mothers using a large national database.

- We used survey-based statistical analyses, based on the design-effect related to survey sampling.

- Various health behavioural factors of adolescents and mothers were adjusted.

- There is no causal relationship as this was a cross-sectional study.

- The study did not provide any information on nutritional factors which could be significant confounders.

all ages. ${ }^{1}$ In Korea, CVD is the second-leading cause of death after cancer. ${ }^{2}$ Triglyceride (TG) and high-density lipoprotein cholesterol (HDL-C) are the major components of metabolic syndrome (MetS). Likewise, the TG-toHDL-C ratio, a predictor for small, dense, low-density lipoprotein cholesterol (LDL-C), is an independent determinant of arterial stiffness in adolescents and young adults ${ }^{3}$ which can subsequently accelerate atherosclerosis and increase cardiovascular events in the second decade of life. ${ }^{4}$ Meanwhile, lipid level is strongly linked to the body mass index (BMI) which is one of the reliable indicators for obesity in adolescents. ${ }^{5}$ Paediatric obesity is affected by various family settings such as eating habits, lifestyle and education. ${ }^{6}$ The prevalence of paediatric obesity in South Korea has been increasing rapidly from $5.8 \%$ in 1997 to $11.5 \%$ in $2014,{ }^{7}$ which is close to the $13.3 \%$ in the USA. ${ }^{8}$ This has increased interest in obesity-related disorders in adolescents, such as metabolic, cardiovascular and psychosocial complications. ${ }^{9}$ Obesity and dyslipidaemia are no longer the problems of adults alone; therefore, adequate screening and control of dyslipidaemia in adolescents has become important in South Korea. 
In addition to obesity, various factors such as physical activity, economic status, education level, nutritional and dietary factors, sleep duration and psychiatric problems, among others, have been associated with lipid concentration. ${ }^{10-12}$ Meanwhile, family histories usually provide important information regarding paediatric diseases. ${ }^{13}$ Regarding the highly heritable traits of dyslipidaemia, several studies showed that there was a close relationship between the lipid concentrations of parents and their offsprings. ${ }^{14-16}$ This familial clustering implies that there may be common denominators, including health behavioural factors within a family, as well as genetic backgrounds. In the present study, we investigated clinical and health behavioural factors affecting adolescents' lipid levels, and evaluated the association between the lipid profiles of adolescents and their mothers.

\section{METHODS}

\section{Data source}

This is a cross-sectional study using the secondary data of the Korea National Health and Nutrition Examination Survey (KNHANES). KNHANES is an ongoing surveillance system conducted by Korea Centers for Disease Control and Prevention (KCDC) since 1998 that assesses health and nutrition status, and monitors health risk factors and the prevalence of chronic diseases. ${ }^{17} \mathrm{~A}$ special survey team visits four regions every week (192 regions per year) and conducts a health examination, health interview and nutrition survey. This survey includes a representative sample of the population selected using a stratified, multi-stage and clustered sampling method. Sampling units are district, survey area and household. Stratification variables are city/province, district and housing type. The sample is weighted to reflect sampling rate, response rate and population demographics in order to estimate health consciousness, health behaviour and nutritional status for the population.

Among 59015 individuals who were surveyed in KNHANES between 2009 and 2015, we selected 4148 adolescents aged 12-18 years with available lipid profile data. Next, we obtained data for the mothers of these adolescents during the same survey period by matching household identification numbers. After the exclusion of 1264 individuals with missing information about adolescent's or mother's baseline characteristics or clinical findings, 2884 adolescents were eligible for the study (figure 1).

\section{Outcome variables and health behavioural factors}

Both adolescents' and mothers' lipid profiles consisted of total cholesterol (TC), TG, HDL-C and LDL-C. Outcome variables in the study were adolescents' lipid levels. Mothers' lipid levels, which represent genetic linkage, were the interesting variables. In order to examine their relationship, we adjusted various clinical and health behavioural factors of both adolescents and mothers. The level of LDL-C was calculated using

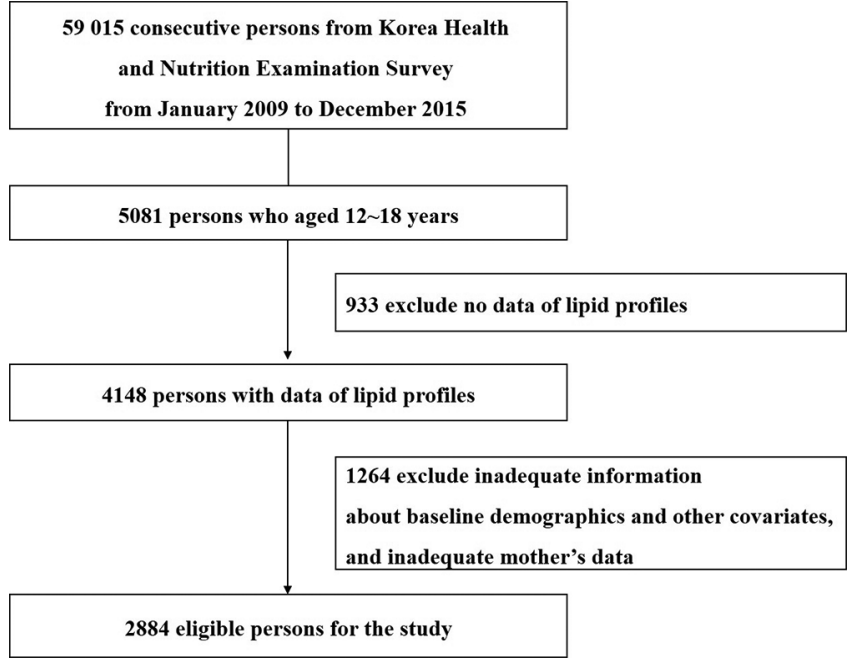

Figure 1 Study flow showing sample selection. We selected 2884 adolescents aged 12-18 whose mothers' data were also available.

the Friedewald equation. If the TG level was $400 \mathrm{mg}$ / $\mathrm{dL}$ or more, measurement of LDL-C was performed by using the immunochemical method. Adolescents were divided into two age-groups based on whether they were high school students. In terms of obesity, we divided the study subjects into two groups using $85 \%$ cut-off of the body mass index (BMI) based on the age-groups and sex for adolescents, and divided them into three groups $\left(<23,23-24.9, \geq 25 \mathrm{~kg} / \mathrm{m}^{2}\right)$ for mothers. ${ }^{18} 19$ The values of fasting glucose were also divided into two groups based on the level of impaired fasting glucose $(\geq 100 \mathrm{mg} / \mathrm{dL})$. Degree of stress was divided into three groups based on individuals' perception. In addition, frequency of eating out, walking and exercise per week were investigated for adolescent health behaviours.

For mothers' variables, we used data regarding smoking and alcohol habits, degree of education and family income, economic activity and frequency of eating out per week. Mother's dyslipidaemia was defined based on TC level of $240 \mathrm{mg} / \mathrm{dL}$ or more, and included cases of individuals diagnosed or treated with dyslipidaemia even if the TC level was normal.

\section{Statistical methods}

Lipid profiles were analysed as continuous variables with mean and SD in both adolescents and their mothers. We checked whether the continuous variables were normally distributed, and used a log-scale depending on the results. Independent sample t-tests or one-way analysis of variances was used for categorical independent variables to analyse the relationship with adolescents' lipid levels. The correlation of lipid levels between adolescents and their mothers was analysed using partial correlations (r) with $95 \%$ CI. The $\mathrm{r}$ values were interpreted as slight $(>0-0.2)$, fair $(>0.2-0.4)$, moderate $(>0.4-0.6)$, substantial $(>0.6-0.8)$ and almost perfect $(>0.8)$. Next, multiple linear regressions with parameter estimates (beta coefficients) and SE were performed to identify the amount 
change in adolescents' lipid levels for each unit increase of their mothers' lipids. We used survey-based statistical regression analyses, and the design-effect relating survey sampling was calculated. The regression models included clinical characteristics and health behavioural factors of both adolescents and mothers. In order to find the most adequate model fits for 16 possible combinations between four adolescents' and their mothers' lipid profiles, we calculated the adjusted $\mathrm{R}^{2}$ values, which represent the explanatory power of the model. In addition, the beta coefficients were also determined in the subgroups by sex and mother's characteristics (age-group, BMI, degree of education, economic activity and presence or absence of dyslipidaemia) using multiple linear regression. Lastly, sensitivity test was done on 4148 adolescents including 1264 subjects who had inadequate baseline information or missing mothers' data to identify the baseline characteristics. All 2 -sided p values $<0.05$ were considered significant. Statistical analyses were performed using SAS V.9.4 (SAS Institute, Cary, NC, USA).

\section{Patient and public involvement}

This study is a population-based survey study. Patients and public were not involved.

\section{RESULTS}

Table 1 shows baseline characteristics and their associations with adolescent lipid levels; $p$ values were calculated considering log-transformed outcome values. The mean age of the study population was $14.7 \pm 1.9$ years (range, 12-18 years), and $52.8 \%$ of the adolescents were male. A total of $9.3 \%$ of the individuals were overweight. The mean levels (ranges) of adolescents' lipids were $156.6 \pm 27.0$ (82-350), 83.6 \pm 46.4 (15-602), 50.4 \pm 9.8 (22-96) and $89.4 \pm 23.3 \mathrm{mg} / \mathrm{dL}(9-296)$, respectively, for TC, TG, HDL-C and LDL-C. HDL-C level was decreased in the older age-group $(\mathrm{p}=0.021)$. While TC, HDL-C and LDL-C levels were significantly higher in female adolescents than in their male counterparts, TG was not different by sex. Individuals with increased BMI showed higher TC, TG and LDL-C levels, and lower HDL-C levels compared with those within the normal percentile range for BMI. The frequency of eating out was inversely associated with TC level ( $\mathrm{p}=0.032)$, while increased frequency of walking was associated with decreased TC and LDL-C levels ( $p=0.006$ and $p=0.005$, respectively). TG levels tended to increase in the adolescents whose mothers were obese $\left(\mathrm{BMI} \geq 25 \mathrm{~kg} / \mathrm{m}^{2}\right.$ ), while the level of HDL-C was inversely associated with the mother's BMI and increasing age. Other health behaviours of the mothers did not show any significant associations with their adolescents' lipid levels.

Adolescent TC level demonstrated a fairly positive correlation with mother's TC level $(r, 0.271 ; 95 \%$ CI 0.236 to 0.304 ) (online supplementary figure $\mathrm{S} 1$ ). TG, HDL-C and LDL-C levels also had fairly positive correlations between adolescents and their mothers, yielding $r$ (95\% CI) 0.204 (0.169 to 0.239 ), 0.289 (0.255 to 0.322 ) and 0.286 (0.252 to 0.319), respectively. For reference, the correlations among the four adolescent lipid profiles demonstrated an almost perfect correlation between the TC and LDL-C levels $(r, 0.915 ; 95 \%$ CI 0.909 to $0.921 ; \mathrm{p}<0.001$ ), and showed a significant negative correlation between HDL-C and TG $(r,-0.329$; $95 \%$ CI -0.361 to $-0.296 ; \mathrm{p}<0.001)$. Meanwhile, the partial correlation coefficient (95\% CI) for TC, TG, HLD-C and LDL-C was 0.254 (0.206 to 0.301 ), 0.235 (0.186 to 0.282 ), $0.271(0.224$ to 0.317$)$ and $0.267(0.220$ to 0.313$)$ in male subjects $(\mathrm{n}=1522)$, and it was $0.291,(0.241$ to 0.339$)$, 0.168 , ( 0.116 to 0.220$), 0.317$ (0.268 to 0.364$)$, and 0.309 $(0.260$ to 0.357$)$ in female subjects $(n=1362)$. All $p$ values were $<0.001$.

Based on the adjusted $\mathrm{R}^{2}$ values, the four most adequate regression models were selected (online supplementary table S1). Table 2 displays the multiple linear regressions of the four adequate models. It appears that $\mathrm{p}$ values are in $\log$ scale. The design-effect from survey sampling was 1.01, 1.43, 1.07 and 1.07 in TC, TG, HDL-Cand LDL-C respectively. Adolescent TC increased by $0.23 \mathrm{mg} / \mathrm{dL}$ on average as their mothers' TC increased by $1 \mathrm{mg} / \mathrm{dL}$ (SE, 0.02, $\mathrm{p}<0.001$ ). The beta coefficients were 0.16 (SE, 0.01), 0.24 (SE, 0.02) and 0.24 (SE, 0.02), respectively, in each model of TG, HDL-Cand LDL-C (all $\mathrm{p}<0.001$ ). TC increased by $13.32 \mathrm{mg} / \mathrm{dL}$ in female adolescents compared with their male counterparts; other lipid parameters were also higher in female adolescents compared with their male counterparts. BMI had a positive association with the levels of TC, TG and LDL-C, while HDL-C was negatively associated with BMI. The frequency of eating out and walking tended to be inversely associated with TC and LDL-C. Exercise for more than 3 days per week was associated with increased TC and LDL-C levels compared with no exercise. With regard to mother's variables, overall adolescents' lipid levels tended to decrease as their mothers' age increased, and other lipids apart from HDL-C tended to decrease when the mother's BMI increased. Mothers' increased alcohol consumption was also significantly associated with adolescents' decreased HDL-C. Mothers' education, working hours, frequency of eating out and family income did not affect adolescent lipid levels.

Figure 2 represents the amount of change in adolescents' lipid levels with each unit increase in mothers' lipids in the subgroups. In most subgroups, there were significant positive relationships between lipids in adolescents and mothers, with the exception of subgroups with relatively small sample sizes (table 3 ). The beta coefficients of TC, HDL-C and LDL-C were high in female adolescents compared with their male counterparts, whereas that of TG was higher in the male adolescents. When the lipid profiles were considered as binary outcomes, multivariate logistic regressions showed that adolescents' dyslipidaemia was significantly associated with mothers' dyslipidaemia (online supplementary table S2). Finally, the sensitivity test on 4148 adolescents showed comparable baseline characteristics with our study data (online supplementary table S3). 


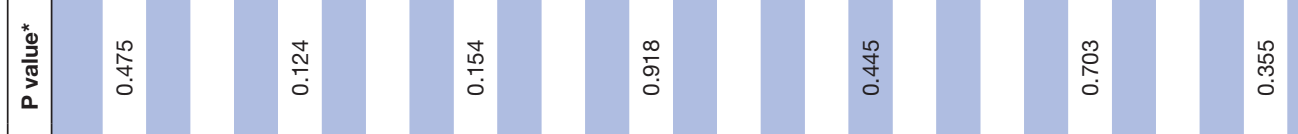

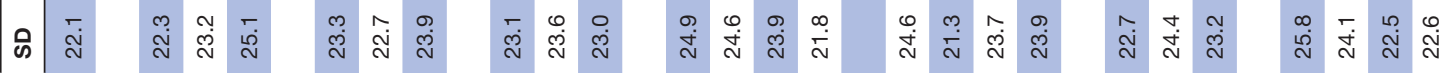

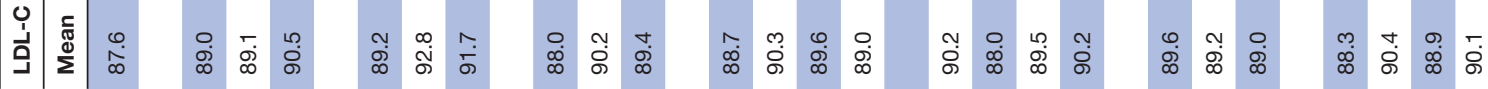

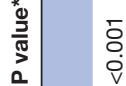

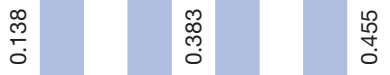

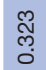
胥

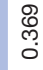

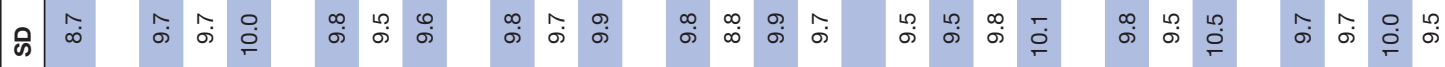

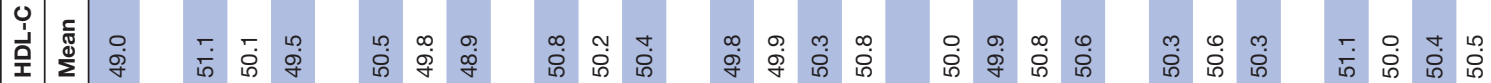

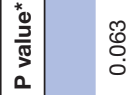

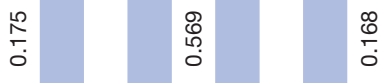
$\stackrel{\stackrel{\circ}{8}}{\circ}$
$\underset{\substack{\infty \\ \infty}}{\infty}$
๕̊:

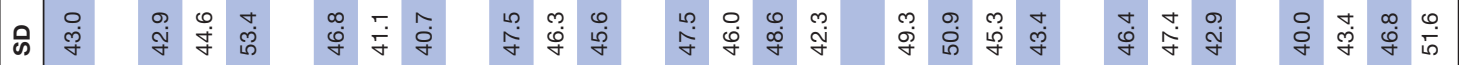

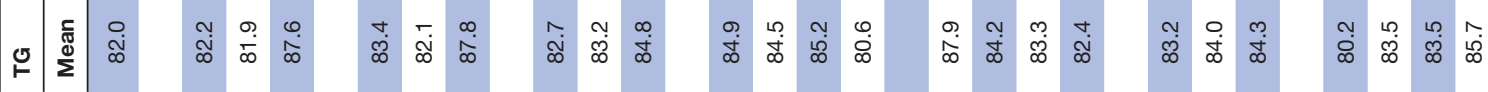

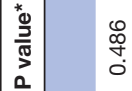

$\underset{\substack{0 \\ 0}}{\substack{0 \\ 0}}$

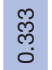

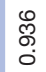

疍

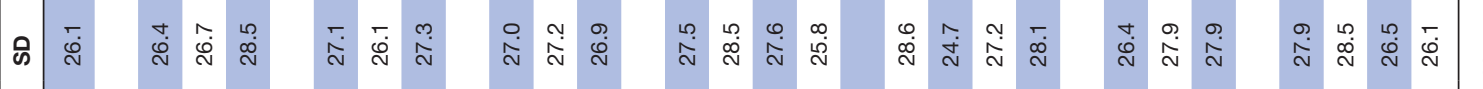

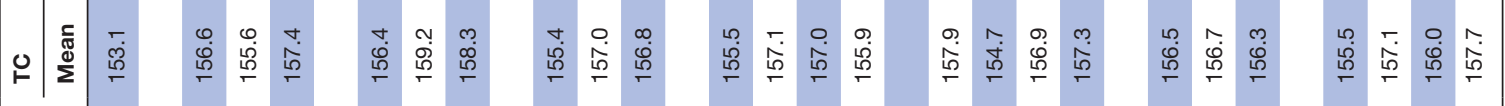

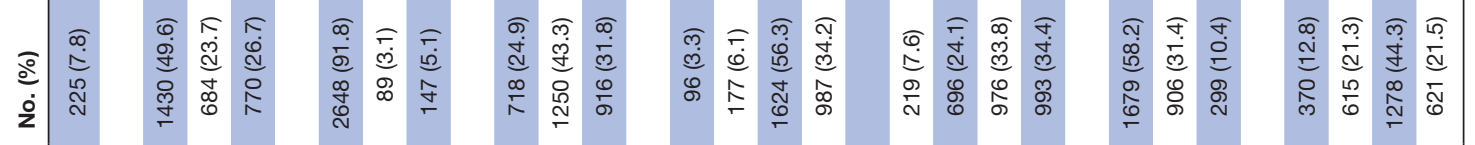

व

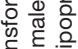

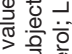

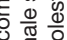

政

可 $\subseteq$

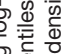

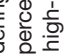

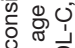




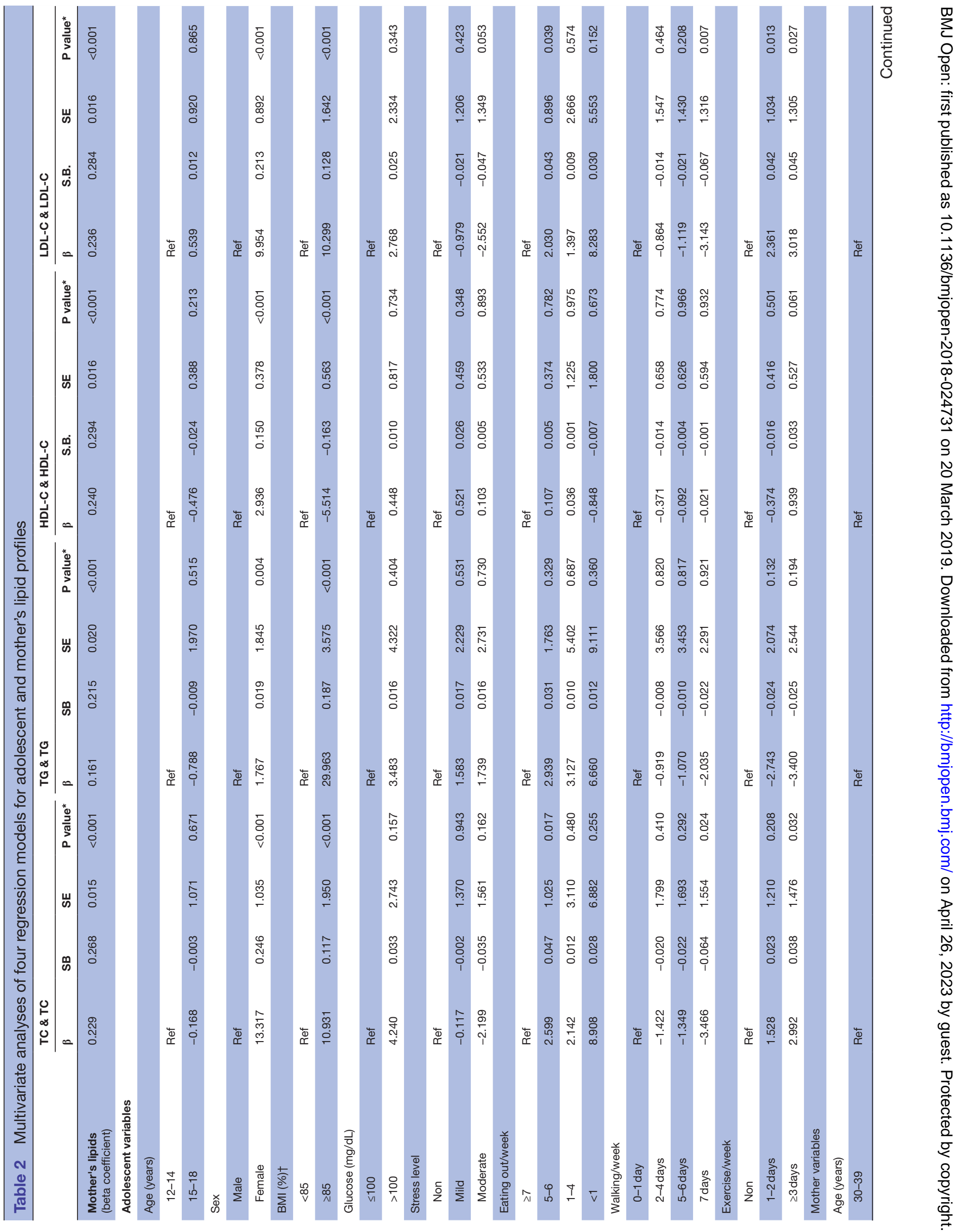




\begin{tabular}{|c|c|c|c|c|c|c|c|c|c|c|}
\hline $\begin{array}{l}\frac{3}{3} \\
\frac{3}{3} \\
0\end{array}$ & 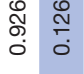 & \begin{tabular}{l}
$\stackrel{2}{0}$ \\
\hdashline \\
0
\end{tabular} & 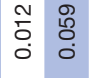 & 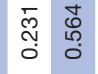 & 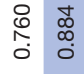 & $\begin{array}{l}\infty \\
\vdots \\
0 \\
0\end{array}$ & $\begin{array}{ll}\hat{m} & \hat{o} \\
0 & 0\end{array}$ & 点 & & 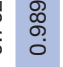 \\
\hline & 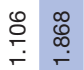 & & & & 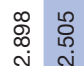 & $\begin{array}{l}\stackrel{\leftrightarrow}{\circ} \\
\stackrel{\circ}{\mathrm{N}}\end{array}$ & 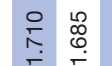 & 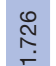 & & s. \\
\hline
\end{tabular}

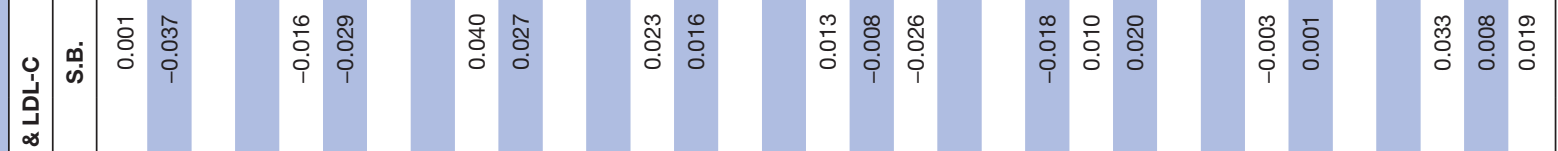

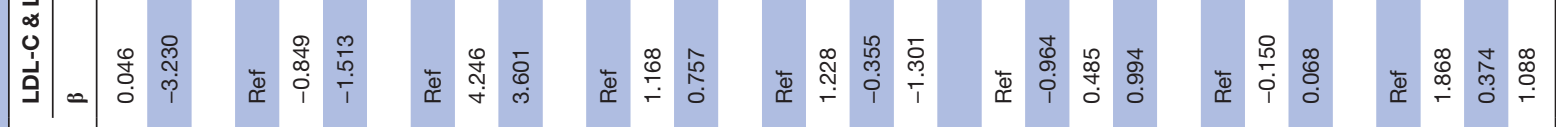

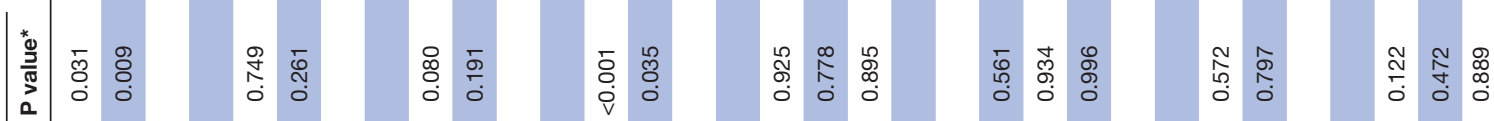

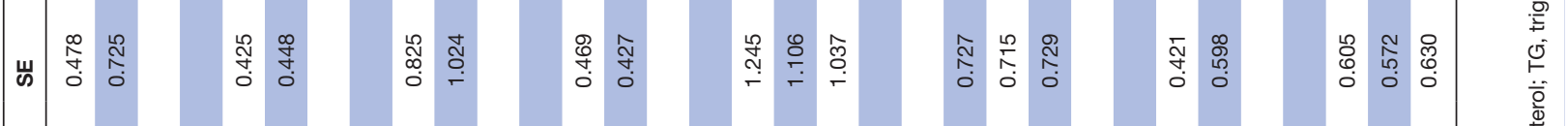

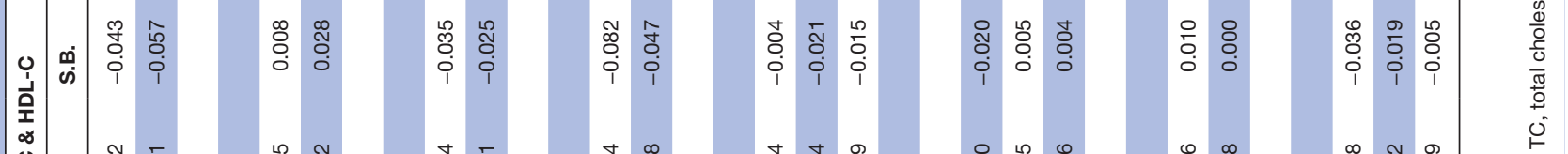

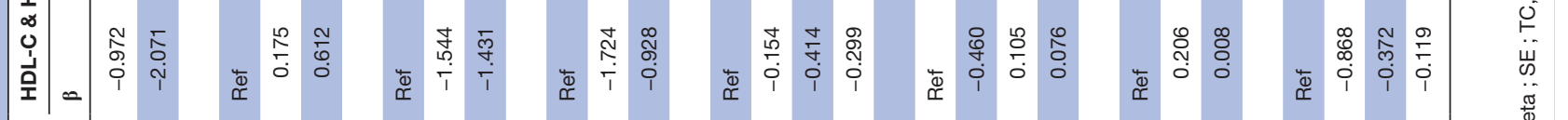

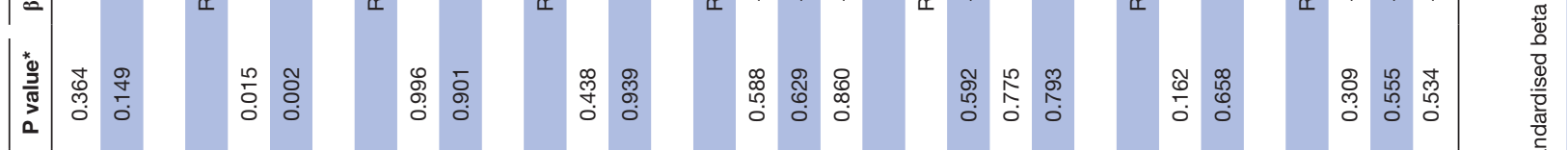

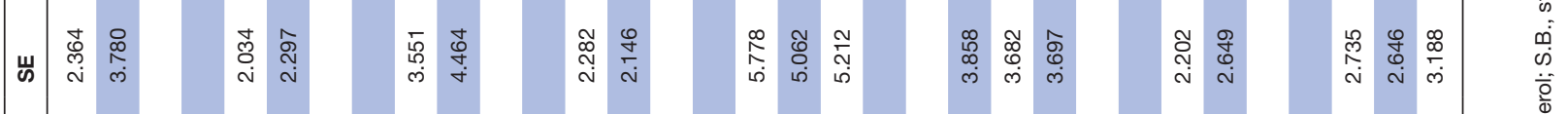

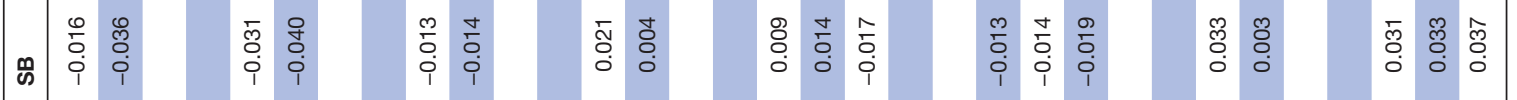

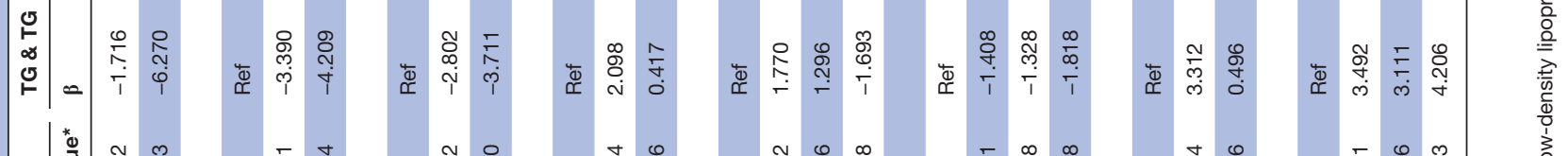

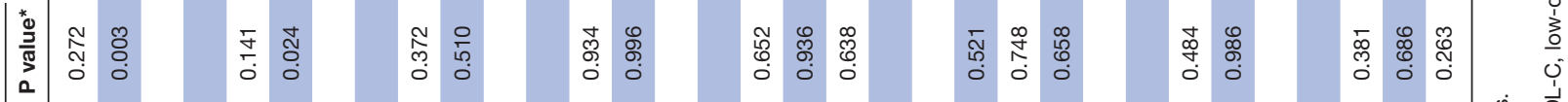

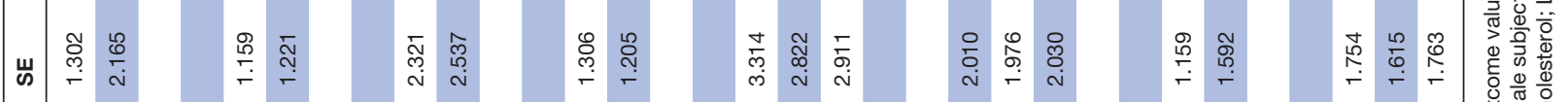

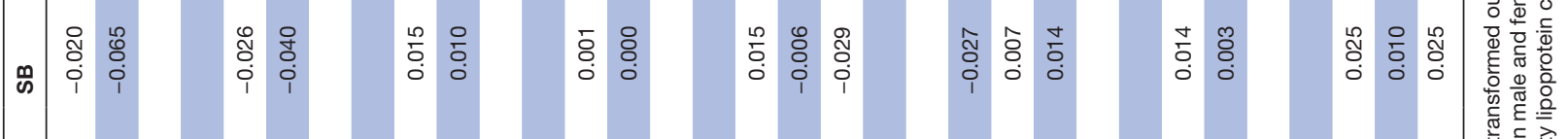

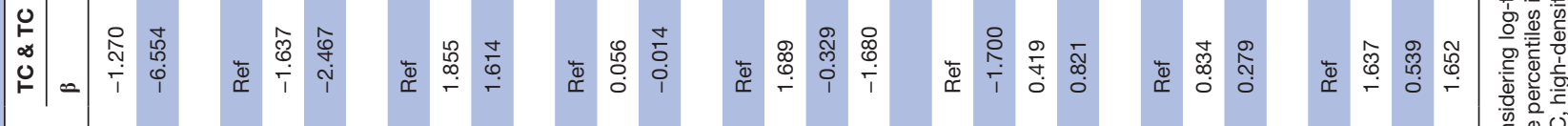

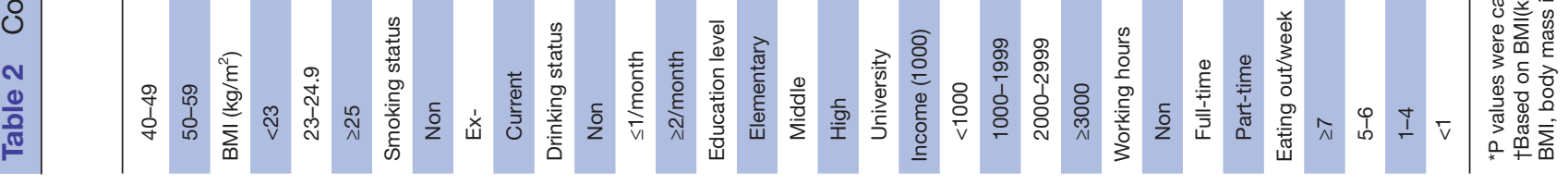
항ㅎㅎㅁㅜ

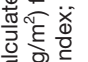



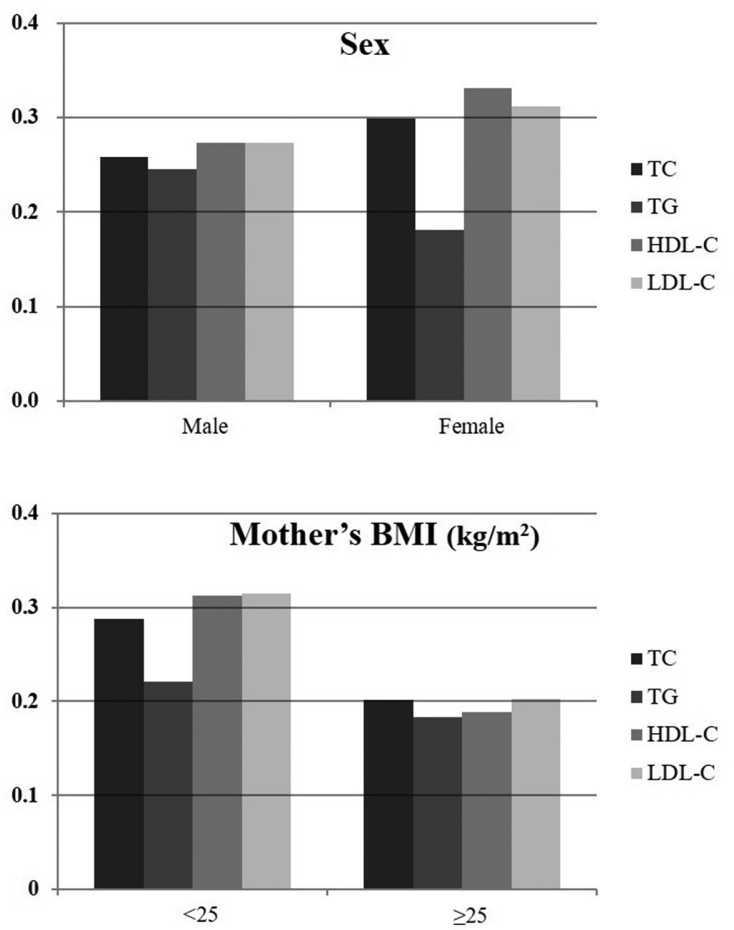
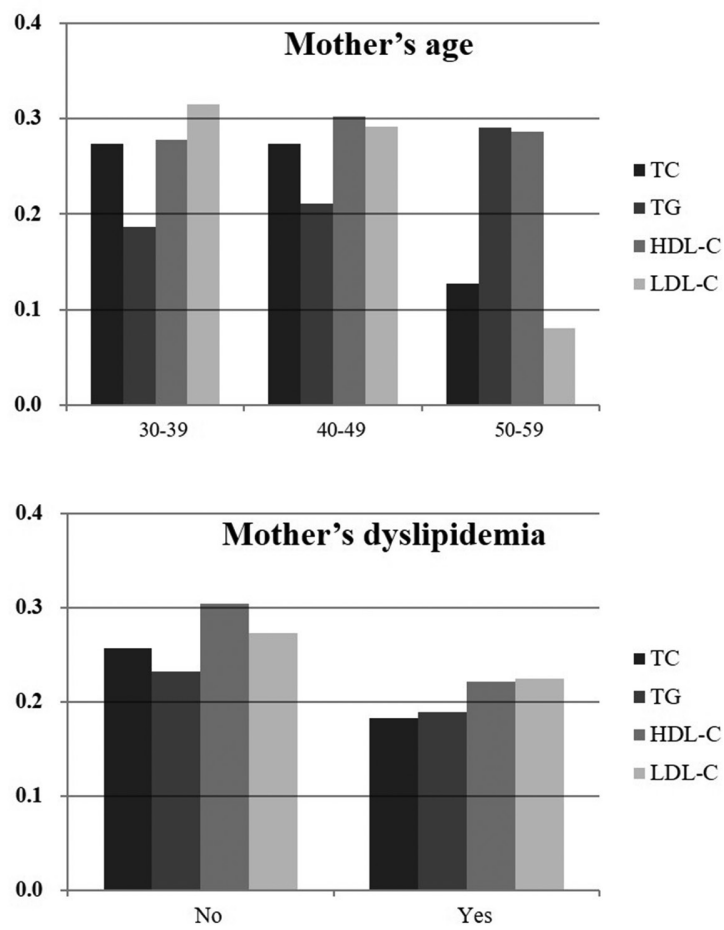

Figure 2 Bar graphs showing standardised beta coefficients of adolescents' lipids for each unit increase in their mothers' lipids in subgroups. BMI, body mass index; HDL-C, high-density lipoprotein cholesterol; LDL-C, low-density lipoprotein cholesterol; TC, total cholesterol; TG, triglyceride.

\section{DISCUSSIONS}

There is significance in that our study analysed linear relationships of TC, TG, HDL-C and LDL-C, respectively, with the amount change in adolescents' lipid levels for each unit increase in their mothers' lipids. We adjusted for various health behavioural factors of adolescents and their mothers, as well as used a large national database. Moreover, we found that relationships between lipids of adolescents and their mothers were significant regardless of sex and mother's characteristics.

Atherosclerosis is triggered by childhood obesity associated with lipid abnormalities, rather than by obesity itself. $^{20}$ The prevalence of dyslipidaemia was $6.5 \%$ in Korea by the cut-off of National Cholesterol Education Programme and American Heart Association guidelines. ${ }^{21}$ Meanwhile, the most frequent components among five MetS criteria in adolescence were high TG $(21.2 \%)$ and low HDL-C $(13.6 \%){ }^{22}$ When cut-off values of a recent guideline were applied to our data, ${ }^{23}$ the percentages of abnormal TC ( $\geq 200 \mathrm{mg} / \mathrm{dL})$, TG $(\geq 130 \mathrm{mg} / \mathrm{dL})$, HDL-C $(<40 \mathrm{mg} / \mathrm{dL})$ and LDL-C $(\geq 130 \mathrm{mg} / \mathrm{dL})$ were $6.6 \%$, $11.9 \%, 13.3 \%$ and $5.0 \%$, respectively. Atherogenic dyslipidaemia, characterised by the combination of high TG and small dense LDL-C and low HDL-C, was a common form of dyslipidaemia in young individuals (aged, 2-18 years) and had a strong familial aggregation. ${ }^{24}$ Even taking into consideration the argument that a higher cut-off level of TG $(\geq 150 \mathrm{mg} / \mathrm{dL})$ is appropriate for Korean adolescents, ${ }^{25}$ the rate of high TG observed in the present study was $7.7 \%$. That is, our data showed, a more considerable proportion of abnormal TG and HDL-C in adolescents compared with other lipid parameters. Thus, the present study provides further evidence that dyslipidaemia, especially atherogenic dyslipidaemia, is a big problem in Korean adolescents, with the concern that it leads to CVD during the remainder of the lifespan.

It has been reported that dyslipidaemia was associated with increased odds of dyslipidaemia in first-degree relatives $(\mathrm{OR}=2.2){ }^{26}$ This familial clustering is, in turn, caused by both genetic backgrounds and shared environmental factors within a family. A previous study found that genes contribute more than the environment to familial correlation of lipids and obesity. ${ }^{15}$ In this regard, numerous genetic determinants regulating lipid concentrations have been investigated. ${ }^{27}$ In addition, an animal study demonstrated that maternal dyslipidaemia affected the offspring's lipid levels by activation of endogenous cholesterol synthesis. ${ }^{28}$ Whatever the cause or, a family history must be a major risk factor for adolescent's dyslipidaemia. Meanwhile, even in the subgroup of mothers who had normal TC levels and had never been diagnosed with dyslipidaemia, the positive relationships in lipids between the adolescents and their mothers were significant for all lipid parameters. These findings may reflect environmental impacts such as healthy diet, exercise habits, and efforts to improve lifestyles within families, rather than just a hereditary influence. Of course, there may also be an impact from other genetic factors such as diabetes or hypertension in first-degree relatives. ${ }^{26}$ Interestingly, the beta coefficient was higher in adolescents with non-obese mothers compared with those with obese mothers. It is possible that the genetic background of 


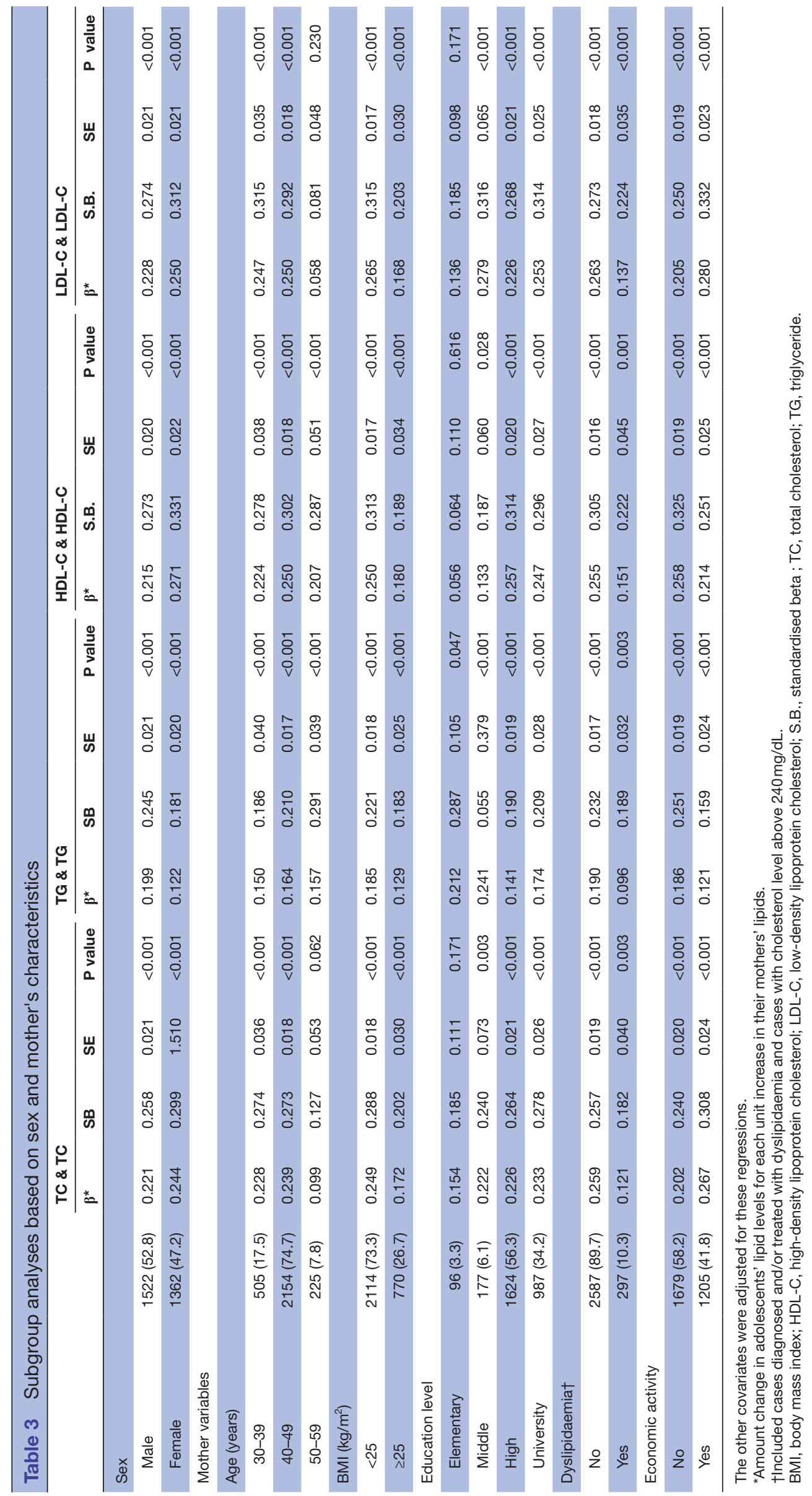


non-obese dyslipidemic mothers affected the lipid levels of their offspring. However, the mean BMI of dyslipidemic mothers was higher than that of non-dyslipidemic mothers $\left(24.7 \mathrm{~kg} / \mathrm{m}^{2}\right.$ vs $\left.23.2 \mathrm{~kg} / \mathrm{m}^{2}\right)$. Moreover, the beta coefficient was also higher in adolescents with non-dyslipidemic mothers than in those with dyslipidemic mothers. Thus, it is more likely that the mothers' perception of dyslipidaemia influences the adolescents' lipid levels. Of course, this interpretation requires consideration of relationship between lipids and characteristics in mothers. Awareness of dyslipidaemia was relatively low despite its higher prevalence worldwide. ${ }^{29} \mathrm{~A}$ mother's perception of lipid levels could affect her children's lipids through efforts related to lifestyle and diet changes. ${ }^{30}$ A recent Korean study highlighted education and counselling in order to change health behaviour in addition to awareness of dyslipidaemia. ${ }^{31}$ Our results from subgroup analyses support these previous studies and highlight the influence of the mother's perception of dyslipidaemia and resultant lifestyle changes.

There is no doubt that lifestyle modification plays a central role in lipid control. Moreover, considering the high rates of abnormal TG and HDL-C and the restricted indications of lipid-lowering agents in youth, lifestyle changes should play a larger role in adolescent patients. Our results showed that frequent walking was negatively associated with TC and LDL-C levels, which is predictable. Meanwhile, frequent eating out was associated with decreased TC and LDL-C, a finding that conflicts with the general notion that eating out induces a high calorie intake or overeating. Eating out was defined as all foods except home-cooked dishes in this survey, thus including school meals as well as dining out and delivery foods. Actually, the frequency of eating out showed a great discrepancy between adolescents and mothers in this study. Thus, school foods may compensate for negative effects of eating out by providing regular and well-balanced meals. The positive correlation between exercise and lipid levels, which is also an unexpected result, seems to be influenced by exercise intensity. Exercise frequency alone was not sufficient to explain the effect of exercise adequately; thus, the strength and duration of exercise should be considered. Our data regarding health behavioural factors should be more detailed and concrete. However, it is certain that health behavioural habits influence the lipid levels of adolescents, and therefore adolescents with dyslipidaemia and their families should be encouraged to improve their lifestyles.

Cholesterol levels in children and adolescents are highly dependent on age and sex. ${ }^{32}$ Our data showed that the levels of TC, LDL-C and HDL-C were higher in female adolescents that in males. In addition, the beta coefficients per unit increase of mother's TC, LDL-C and HDL-C were also prominent in female subjects. It is possible that mothers with female offspring are either more obese and dyslipidemic or otherwise. However, mother's mean BMI was similar between male and female adolescents $\left(23.3 \pm 3.2\right.$ and $23.5 \pm 3.3 \mathrm{~kg} / \mathrm{m}^{2}$, respectively, $\mathrm{p}=0.161$ ); furthermore, the rate of mother's dyslipidaemia showed no statistical difference between male and female adolescents $(10.8 \%$ vs $9.8 \%$, respectively, $\mathrm{p}=0.373)$. Thus, the difference in beta coefficient by sex may be due to a distinct difference in lipid levels by sex. This is supported by our result that the TG level was higher in male than in female adolescents and the beta coefficient of TG was also higher in male adolescents.

This study has several limitations. First, because it is a survey-based study, our data are vulnerable to recall bias. Second, as it is a cross-sectional design, there was no causal relationship. This factor will be particularly important in consideration of the impacts due to environmental factors. Further well-designed cohort studies are warranted. Third, individuals who responded to the national survey could have greater health concerns. They may have better health behavioural habits or family members with chronic diseases. However, this survey was uniformly performed in all regions of Korea and targeted all age-groups; thus, our data can be considered nationally representative. Fourth, the nutritional factors, which were not considered in the analyses because of insufficient information and large missing values, can be significant confounding factors. Further studies based on detailed surveys for health behavioural factors and nutritional elements are needed. Fifth, we did not evaluate the father's lipid levels. If the father's lipid levels had also been considered, the genetic backgrounds of lipids might be emphasised more. Sixth, various comorbidities such as hypothyroidism, Cushing's disease, liver disease and nephrotic syndrome, among others, as well as longterm use of steroid can affect lipid level, ${ }^{33}$ and these could be also confounding factors. However, these chronic diseases are extremely rare during the adolescent period, and thus could be negligible. Finally, the results of our study need to be evaluated with caution as they might be vulnerable to family-wise type I error due to the multiple tests involved in our analysis. However, even considering this, the $p$ values for the associations are sufficiently significant. Additionally, $\mathrm{R}^{2}$ indicates just how well the model explains variability of the response data. Although we chose four models, which showed high $\mathrm{R}^{2}$, it does not mean accurate representation of goodness of fit for the models.

In conclusion, a mother's lipid levels were positively associated with her adolescents' lipid levels because of both genetic and environmental factors within the family. Adolescent dyslipidaemia creates a large risk factor burden for cardiovascular diseases; therefore, timely screening for dyslipidaemia is important, especially for indicated adolescents. Our positive correlation between lipids of adolescents and their mothers supports that the mother's lipid level is an appropriate reference for the screening of the adolescent's dyslipidaemia.

Author affiliations

${ }^{1}$ Department of Internal Medicine, Dongguk University Ilsan Hospital, Dongguk University College of Medicine, Goyang, Korea (the Republic of) 
${ }^{2}$ Department of Medicine, Graduate School, Yonsei University, Seoul, Korea (the Republic of

${ }^{3}$ Department of Preventive Medicine and Institute of Health Services Research, Yonsei University College of Medicine, Seoul, Korea (the Republic of)

${ }^{4}$ Department of Pediatrics, Dongguk University Ilsan Hospital, Dongguk University College of Medicine, Goyang, Korea (the Republic of)

${ }^{5}$ Division of Cancer Management Policy, National Cancer Center, Goyang, Korea (the Republic of)

Acknowledgements The authors thank the participants for their cooperation and the staffs of KNHANES (https://knhanes.cdc.go.kr/knhanes/index.do) for their hard work.

Contributors ECP and SIJ designed the study. JHN and JS analysed and interpreted the data. JHN, JKL, and YJL drafted the manuscript. JHK and KTH critically revised the manuscript. All authors read and approved the final version.

Funding The authors have not declared a specific grant for this research from any funding agency in the public, commercial or not-for-profit sectors.

Competing interests None declared.

Patient consent for publication Not required.

Ethics approval This study was analyzed using KNHANES secondary data. Use of the data from KNHANES was approved by the Institutional Review Board of the KCDC (2009-01CON-03-2C, 2010-02CON-21-C, 2011-02CON-06-C, 2012-01EXP-01-2C, 2013-07C0N-03-4C, and 2013-12EXP-03-5C). This survey has been available for use without approval since 2015 .

Provenance and peer review Not commissioned; externally peer reviewed.

Data sharing statement All data analysed during this study are available in the KCDC and KNHANES repository, [https://knhanes.cdc.go.kr/knhanes/sub03/sub03_ 01.do].

Open access This is an open access article distributed in accordance with the Creative Commons Attribution Non Commercial (CC BY-NC 4.0) license, which permits others to distribute, remix, adapt, build upon this work non-commercially, and license their derivative works on different terms, provided the original work is properly cited, appropriate credit is given, any changes made indicated, and the use is non-commercial. See: http://creativecommons.org/licenses/by-nc/4.0/.

\section{REFERENCES}

1. Berenson GS, Srinivasan SR, Bao W, et al. Association between multiple cardiovascular risk factors and atherosclerosis in children and young adults. The Bogalusa Heart Study. N Engl J Med 1998;338:1650-6.

2. Shin H-Y, Lee J-Y, Song J, et al. Cause-of-death statistics in the Republic of Korea, 2014. J Korean Med Assoc 2016;59:221-32.

3. Urbina EM, Khoury PR, McCoy CE, et al. Triglyceride to HDL-C ratio and increased arterial stiffness in children, adolescents, and young adults. Pediatrics 2013;131:e1082-90.

4. McGill HC, McMahan CA, Zieske AW, et al. Associations of coronary heart disease risk factors with the intermediate lesion of atherosclerosis in youth. The Pathobiological Determinants of Atherosclerosis in Youth (PDAY) Research Group. Arterioscler Thromb Vasc Biol 2000;20:1998-2004.

5. Margolis KL, Greenspan LC, Trower NK, et al. Lipid screening in children and adolescents in community practice: 2007 to 2010. Circ Cardiovasc Qual Outcomes 2014;7:718-26.

6. Smetanina N, Albaviciute E, Babinska V, et al. Prevalence of overweight/obesity in relation to dietary habits and lifestyle among 7-17 years old children and adolescents in Lithuania. BMC Public Health 2015;15:1001.

7. Korea Centers for Disease Control and Prevention. Korea Health Statistics 2014: Korea National Health and Nutrition Examination Survey (KNHANES VI-2). Seoul, Korea: Ministry of Health and Welfare, 2014.

8. Ogden CL, Carroll MD, Curtin LR, et al. Prevalence of high body mass index in US children and adolescents, 2007-2008. JAMA 2010;303:242-9.

9. Ha KH, Kim DJ. Epidemiology of Childhood Obesity in Korea. Endocrinol Metab 2016;31:510-8.

10. Wang CJ, Li YQ, Wang L, et al. Development and evaluation of a simple and effective prediction approach for identifying those at high risk of dyslipidemia in rural adult residents. PLoS One 2012; $7: e 43834$

11. Danese A, Moffitt TE, Harrington $\mathrm{H}$, et al. Adverse childhood experiences and adult risk factors for age-related disease: depression, inflammation, and clustering of metabolic risk markers. Arch Pediatr Adolesc Med 2009;163:1135-43.

12. Buitrago-Lopez A, van den Hooven EH, Rueda-Clausen CF, et al. Socioeconomic status is positively associated with measures of adiposity and insulin resistance, but inversely associated with dyslipidaemia in Colombian children. J Epidemiol Community Health 2015;69:580-7

13. Tarini BA, Mclnerney JD. Family history in primary care pediatrics. Pediatrics 2013;132:S203-10.

14. Kathiresan S, Manning AK, Demissie S, et al. A genome-wide association study for blood lipid phenotypes in the Framingham Heart Study. BMC Med Genet 2007;8(Suppl 1):S17.

15. Hunt SC, Hasstedt SJ, Kuida H, et al. Genetic heritability and common environmental components of resting and stressed blood pressures, lipids, and body mass index in Utah pedigrees and twins. Am J Epidemiol 1989;129:625-38.

16. Predazzi IM, Sobota RS, Sanna S, et al. Sex-Specific Parental Effects on Offspring Lipid Levels. J Am Heart Assoc 2015;4:e001951.

17. Kweon S, Kim Y, Jang MJ, et al. Data resource profile: the Korea National Health and Nutrition Examination Survey (KNHANES). Int J Epidemiol 2014;43:69-77.

18. Park HW, Yoo HY, Kim CH, et al. Reference values of body composition indices: the Korean National Health and Nutrition Examination Surveys. Yonsei Med J 2015;56:95-102.

19. Jee SH, Sull JW, Park J, et al. Body-mass index and mortality in Korean men and women. N Engl J Med 2006;355:779-87.

20. D'Adamo E, Guardamagna O, Chiarelli F, et al. Atherogenic dyslipidemia and cardiovascular risk factors in obese children. Int $J$ Endocrinol 2015;2015:1-9.

21. Lim JS. The current state of dyslipidemia in Korean children and adolescents and its management in clinical practice. Ann Pediatr Endocrinol Metab 2013;18:1-8.

22. Kim S, So WY. Prevalence of Metabolic Syndrome among Korean Adolescents According to the National Cholesterol Education Program, Adult Treatment Panel III and International Diabetes Federation. Nutrients 2016;8:588.

23. Expert Panel on Integrated Guidelines for Cardiovascular Health and Risk Reduction in Children and AdolescentsNational Heart, Lung, and Blood Institute. Expert panel on integrated guidelines for cardiovascular health and risk reduction in children and adolescents: summary report. Pediatrics 2011;128(Suppl 5):S213-56.

24. Montali A, Truglio G, Martino F, et al. Atherogenic dyslipidemia in children: evaluation of clinical, biochemical and genetic aspects. PLoS One 2015;10:e0120099.

25. Yoon JM. Dyslipidemia in children and adolescents: when and how to diagnose and treat? Pediatr Gastroenterol Hepatol Nutr 2014;17:85-92.

26. Khoury M, Manlhiot C, Gibson D, et al. Universal screening for cardiovascular disease risk factors in adolescents to identify highrisk families: a population-based cross-sectional study. BMC Pediatr 2016;16:11.

27. Calandra S, Tarugi P, Speedy HE, et al. Mechanisms and genetic determinants regulating sterol absorption, circulating LDL levels, and sterol elimination: implications for classification and disease risk. $J$ Lipid Res 2011;52:1885-926.

28. Goharkhay $\mathrm{N}$, Tamayo EH, Yin $\mathrm{H}$, et al. Maternal hypercholesterolemia leads to activation of endogenous cholesterol synthesis in the offspring. Am J Obstet Gynecol 2008;199:273.e1-6.

29. Zhang FL, Xing YQ, Wu YH, et al. The prevalence, awareness, treatment, and control of dyslipidemia in northeast China: a population-based cross-sectional survey. Lipids Health Dis 2017;16:61.

30. Price $\mathrm{JH}$, Casler SM. African-American mothers' perceptions of cholesterol and its effects on their children. J Natl Med Assoc 1996;88:145-50.

31. Cho IY, Park HY, Lee K, et al. Association Between the Awareness of Dyslipidemia and Health Behavior for Control of Lipid Levels Among Korean Adults with Dyslipidemia. Korean J Fam Med 2017;38:64-74.

32. Skinner AC, Steiner MJ, Chung AE, et al. Cholesterol curves to identify population norms by age and sex in healthy weight children. Clin Pediatr 2012;51:233-7.

33. Vodnala D, Rubenfire M, Brook RD. Secondary causes of dyslipidemia. Am J Cardiol 2012;110:823-5. 